\title{
Quantitative assessment of x-ray fluorescence holography for bcc Fe as a test case
}

\author{
T. Hiort, D. V. Novikov, E. Kossel, and G. Materlik \\ Hamburger Synchrotronstrahlungslabor HASYLAB am Deutschen Elektronen-Synchrotron DESY, Notkestrasse 85, \\ D-22603 Hamburg, Germany
}

(Received 16 July 1999; revised manuscript received 23 September 1999)

\begin{abstract}
We report a pure direct X-ray-fluorescence holography measurement. It allows a straightforward data evaluation and a quantitative analysis of the reconstructed atomic images. The experimental hologram of a bcc Fe single crystal recorded with the Fe $K \alpha, \beta$ lines is presented together with its three-dimensional reconstruction. An isotropic spatial resolution is obtained by using the symmetry information from the measured Kossel lines. The fluorescence emitter atom images its neighbors in a volume of $(7 \AA)^{3}$. The measured distances to the neighbors and the spatial resolution agree with the values obtained from calculated holograms within the errors of $\pm 0.2 \AA$.
\end{abstract}

\section{INTRODUCTION}

In 1996 two methods ${ }^{1,2}$ for structure determination with atomic resolution based on the principle of $\mathrm{x}$-ray holography ${ }^{3}$ were experimentally realized. In the direct method, X-ray-fluorescence holography (XFH), ${ }^{1}$ the reference wave from a fluorescing source atom is scattered from neighboring atoms, forming object waves. The interference field intensity of reference and object waves is detected in the far field. Such holographic interference patterns contain phase information and can be used to reconstruct the atomic environment of the source atom. In the reciprocal method, named multiple energy $\mathrm{x}$-ray holography $(\mathrm{MEXH}),{ }^{2}$ the holographic reference wave is provided by a monochromatic beam incident on a sample from an external x-ray source. This wave is scattered elastically from atoms within the sample thus producing a three-dimensional standing-wave pattern. ${ }^{4}$ Its field intensity is registered through the fluorescence yield of a detector atom incorporated in the structure.

In all $\mathrm{X}$-ray holography experiments performed so far $^{1,2,5-13}$ the atoms within a sample are excited to fluorescence by incident monochromatic $x$ rays. Thus, two standing-wave fields, one at the energy of the incident wave and another at the energy of the fluorescence wave, are formed. For MEXH, being based on the interference at the energy of the incident radiation, the interference field contribution of the fluorescence waves should be kept constant in the detected signal. Experimental solutions for pure MEXH would be to integrate the fluorescence yield over the $4 \pi$ solid angle, or to keep the detector system stationary in space with respect to the sample. ${ }^{7}$ The sample has to be rotated relative to the incident beam to measure the angular dependent hologram. XFH requires, consequently, that the interference pattern due to the incident wave is held constant, for example by keeping the incident beam stationary with respect to the sample. In this case, the interference pattern has to be recorded by a detector which moves around the sample in the scattering far field. In all previous x-ray holography measurements, these requirements for the experimental setup were not strictly fulfilled. ${ }^{1,2,5,6,8-13}$ This resulted in so-called mixed holograms which contain information from XFH and from $\mathrm{MEXH}^{5,8,14}$ and leads to systematic artefacts in the reconstructions.

\section{EXPERIMENTAL SETUP}

The experiment was performed at the x-ray undulator beamline BW1 at the Hamburg synchrotron radiation laboratory HASYLAB. ${ }^{15,5}$ The photon beam from the DORIS storage ring is focused with two $\mathrm{Au}$ coated mirrors. The energy of $9.0 \mathrm{keV}$ is selected by a $\mathrm{Si}(111)$ double crystal monochromator and the beam is limited by slits to 1 $\times 1 \mathrm{~mm}^{2}$. The incident intensity is monitored by an ionization chamber. An Fe single crystal with (001) surface orientation was chosen as an object, because all atoms have the same configuration of neighboring atoms in the Fe bcc structure with lattice constant $a=2.87 \AA .{ }^{16}$ The emitted fluorescence radiation is measured by two silicon drift detectors with an energy resolution of $\sim 300 \mathrm{eV}$. Fe $K \alpha$ and $K \beta$ lines of wavelengths $\lambda=1.94$ and $1.75 \AA$, respectively, were recorded simultaneously. The results obtained at the two different wavelengths were combined to obtain a more reliable result. ${ }^{17}$ Sample and detectors were mounted on a multicircle diffractometer. The sample was fixed relative to the incident beam and the detectors were rotated around the sample in $1^{\circ}$ angular steps in elevation $(\alpha)$ between sample surface plane and detector window normal and in azimuth $(\varphi)$. Both detectors covered the full $2 \pi$ range in $\varphi, \alpha$ ranging from $0^{\circ}$ to $59^{\circ}$ for one detector and from $24^{\circ}$ to $83^{\circ}$ for the other detector. Due to geometric shading from the sample holder, only the $\alpha$ range from $\sim 18^{\circ}$ to $83^{\circ}$ was used in the holograms. The angular resolution of $\sim 1^{\circ}$ results from detector acceptance angle and illuminated sample area.

The incident flux of $5 \times 10^{10}$ photons/s lead to an average count rate of $\sim 10^{4}$ photons/s in each detector, accumulating to about $10^{10}$ photons in the complete data set in a measuring time of 6 days.

\section{PURE EXPERIMENTAL XFH HOLOGRAMS}

A hologram is formed by the interference of a reference wave $R(\mathbf{k})$, which is the direct fluorescence wave in $\mathrm{XFH}$, 


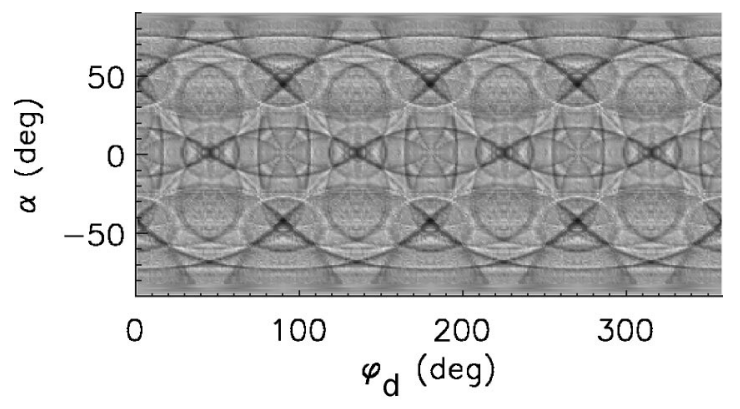

FIG. 1. The hologram obtained by extending the sum over all holograms, measured at the $\mathrm{Fe} K \alpha$ line by one detector, to the symmetrically equivalent parts of the full sphere.

with object waves $O(\mathbf{k})$, $\mathbf{k}$ being the wave vector. The observed intensity is given by

$$
I(\mathbf{k})=|R(\mathbf{k})+O(\mathbf{k})|^{2}=|R|^{2}+R^{*} O+R O^{*}+|O|^{2} .
$$

Terms of the order $|O(\mathbf{k})|^{2}$ and higher can be neglected for clusters and for crystals far away from Bragg angles. This is justified by the small photon-electron scattering cross sections at $\mathrm{x}$-ray wavelengths. ${ }^{18}$ The holographic interference term is given by the mixed terms on the right side of Eq. (1) and can be obtained in a normalized form via

$$
\chi(\mathbf{k}) \simeq \frac{I(\mathbf{k})-|R(\mathbf{k})|^{2}}{|R(\mathbf{k})|^{2}} .
$$

The measured intensities $I(\mathbf{k})$ change considerably with $\alpha$ due to varying $\mathrm{x}$-ray-absorption conditions. $|R(\mathbf{k})|^{2}$ was thus determined by straightforward fitting of an angular dependent absorption correction. Filtering as used, e.g., in Ref. 13 is not applied to the data.

The Kossel lines ${ }^{19}$ visible in the holograms (Fig. 1) (Refs. $5,6)$ clearly show the four-fold symmetry of the structure along $\varphi$. Due to the properly chosen angular resolution of $\sim 1^{\circ}$ no single extrema dominate the holograms so that no Kossel lines were removed. The Kossel lines offer a way to find symmetry elements of the crystal structure, which can be used to extend the measured holograms to symmetrically equivalent parts of the full sphere. This extension reduces significantly the statistical fluctuations of the data set and thus emphasizes the holographic signal. It also avoids truncation artifacts in the reconstruction due to edges in the hologram. The extended sum over all holograms obtained for one detector at the $\mathrm{Fe} K \alpha$ line is shown in Fig. 1.

\section{ATOMIC IMAGES}

The holographic signal $\chi(\mathbf{k})$ is converted to an atomic image $|u(\mathbf{r})|^{2}$ as discussed by Barton. ${ }^{20,17}$ In the approximation of a finite, discrete hologram

$$
u(\mathbf{r}) \propto \sum_{k, \alpha, \varphi} \cos (\alpha) \chi(\mathbf{k}) e^{-i(k r-\mathbf{k} \cdot \mathbf{r})} .
$$

The $R^{*} O$ term of Eq. (1) creates images at atom positions $\mathbf{r}_{j}$ while the $R O^{*}$ term creates twin images at positions $-\mathbf{r}_{j}$.

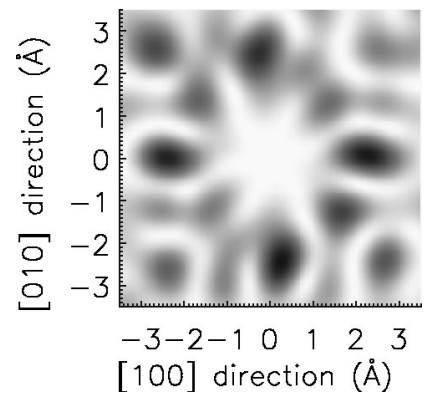

FIG. 2. Reconstructed image obtained from the not extended XFH holograms (not shown). The plane parallel to the sample surface and the (001) lattice planes cutting through the emitter atom at the origin is displayed without background cutoff in a linear grey scale. The dark color corresponds to high peak intensity.

This can lead to uncorrect atom positions at single energies or even to the cancellation of atomic and twin images. ${ }^{20,17,18}$ Pairs of atoms at $\pm \mathbf{r}_{j}$ cannot be imaged when for certain positions $\mathbf{r}_{j}$ the image of the atom at $+\mathbf{r}_{j}$ and the twin image of the atom at $-\mathbf{r}_{j}$ cancel each other. ${ }^{18}$ This can be circumvented using a wide range of energies ${ }^{17}$ as is possible in $\mathrm{MEXH}$. For the XFH of an Fe sample, only the Fe $K \alpha$ and $K \beta$ lines are available so that atoms may be imaged at uncorrect positions even if the reconstructions for both energies are summed using Eq. (3).

The reconstructions were carried out in three dimensions for the unextended hologram according to Eq. (3) in a volume of $(7 \AA)^{3}$. For a data set limited in the $\alpha$ elevation angle range, the best reconstructions are obtained in a plane normal to the $\varphi$ rotation axis. Such a plane parallel to the sample surface and the (001) lattice planes is shown in Fig. 2. The fluorescence emitter atom at the origin does not image itself. The highest maxima in the reconstruction plane appear close to the next-nearest neighbors of the emitter atom in the Fe structure. Weaker maxima occur at the third-nearestneighbor positions. The nearest neighbors of the central fluorescence emitter atom that lie in planes half a lattice constant above and below the image plane are weakly visible. This is due to the limited spatial resolution perpendicular to the sample surface plane. The limited number of counts, possibly a slight misorientation of the sample or an imperfect background removal, disturb the fourfold symmetry in the unextended holograms along $\varphi$. This leads to an imperfect fourfold symmetry in the image plane. Nevertheless, this reconstruction already displays the atomic structure of the image plane well and can be used as a startpoint for a refined structural analysis.

The spatial resolution in the reconstructions depends on the measured angular range of the hologram. For a full $4 \pi$ sphere an isotropic resolution and the minimum full width at half maximum (FWHM) are obtained. The minimum FWHM can be approximated for isotropic scattering by $\lambda \sqrt{2} / \pi,{ }^{21,22}$ yielding $\sim 0.9 \AA$ and $\sim 0.8 \AA$ at the Fe $K \alpha$ and $K \beta$ lines, respectively.

To obtain a three-dimensional image of isotropic spatial resolution, the holograms that were extended to $4 \pi$ were reconstructed. A three-dimensional isosurface at $50 \%$ of the highest maxima is displayed in Fig. 3. The crosspoints of coordinate lines indicate the positions of atoms in the $\mathrm{Fe}$ 


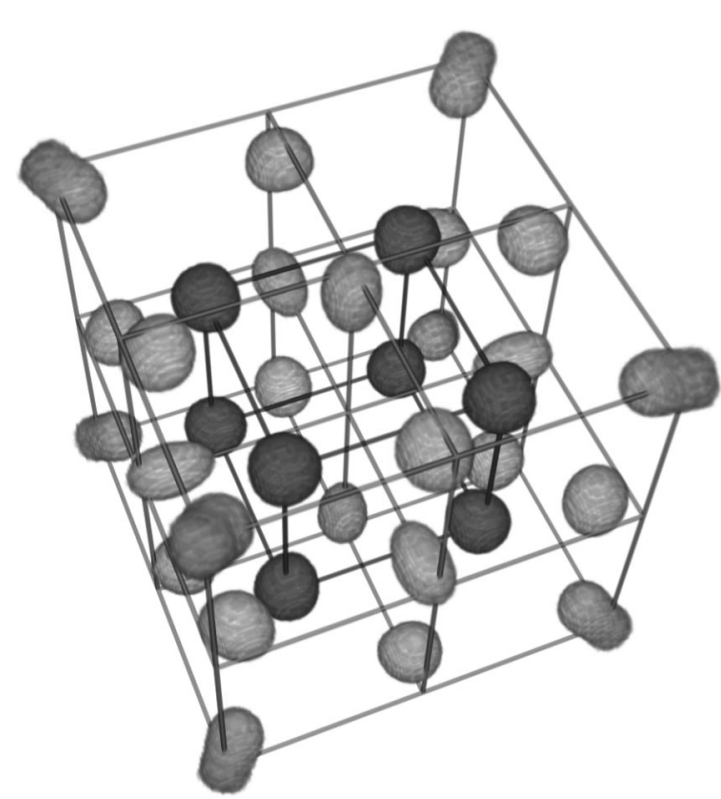

FIG. 3. Three-dimensional nearly isotropic reconstruction of the extended hologram in a volume of $(7 \AA)^{3}$. The nearest neighbors of the emitter atom are displayed in a darker grey than its more distant neighbors. For this plot, the experimental reconstruction was multiplied by the distance from the origin to make atoms at larger distances visible and is displayed with $50 \%$ background cutoff. Atom positions in the Fe lattice are indicated by the crosspoints of coordinate lines.

structure. The emitter atom at the center images the 34 neighbors that are expected from the Fe bcc structure in the imaged volume. The eight nearest neighbors are shown in a darker gray than the 26 more distant neighbors.

The image planes through the fluorescence emitter atom that are parallel to the $\{100\}$ lattice planes look very similar to the plane of Fig. 2 and are not shown. Figure 4(a) shows a plane parallel to the $\{100\}$ lattice planes taken at a distance of $1.4 \AA \approx a / 2$ from the plane containing the emitter atom. It clearly displays the expected four nearest neighbors. For a quantitative analysis, one-dimensional cuts through the three-dimensional reconstruction were evaluated. An example is given in Fig. 4(b). The one-dimensional cut passes through Fig. 4(a) as indicated.

In order to further check the accuracy of the measurement, holograms of a $\mathrm{Fe} \mathrm{bcc}$ cluster of radius $2 a$ were also calculated $^{6,8}$ for the Fe $K \alpha$ and $K \beta$ line energies. Their three-dimensional reconstruction was compared to that of the extended experimental holograms. The atom positions obtained from calculated and measured reconstructions agree with each other within the errors of $\pm 0.2 \AA$. The obtained values for the FWHM vary between $(0.7 \pm 0.2) \AA$ and $(1.2$ $\pm 0.2) \AA$ for different maxima in both reconstructions. These values are close to the theoretical estimate.

As an example, the nearest neighbors of the emitter have a distance of $(2.4 \pm 0.2) \AA$ from the emitter in the reconstruction of the experimental holograms and a distance $(2.2$ $\pm 0.2) \AA$ in the reconstruction of the calculated holograms. Both values are slightly smaller than the tabulated value $a / \sqrt{3}=2.49 \AA$. As another example, the third-nearest neighbors of the emitter have a distance of (3.4 \pm 0.2$) \AA$ from the
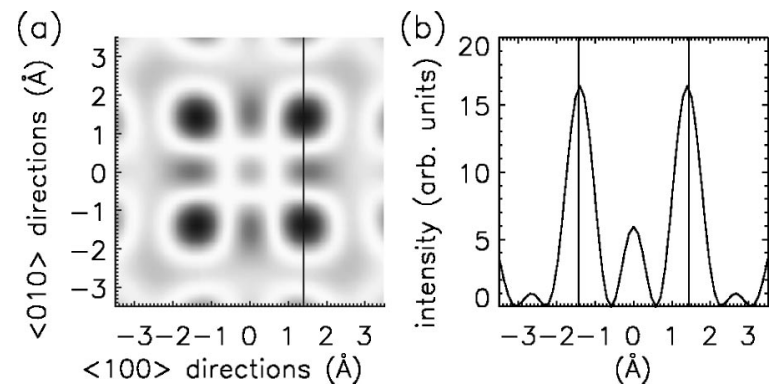

(Å)

FIG. 4. (a) Reconstructed image obtained from the fully extended XFH holograms. The image plane is parallel to the $\{100\}$ lattice planes and taken at a distance of $1.4 \AA$ from the fluorescence emitter atom. The image is displayed without background cutoff in a linear grey scale where dark color corresponds to high peak intensity. (b) One-dimensional cut parallel to the $\langle 100\rangle$ axes as indicated in (a). Vertical lines indicate the atom positions in the Fe structure.

emitter in the reconstruction of the experimental holograms and a distance (3.2 \pm 0.2$) \AA$ in the reconstruction of the calculated holograms. Both values agree within the errors with each other, but they are distinctly smaller than the tabulated $\sqrt{2} a=4.06 \AA$. The calculation thus shows that the shift is mostly due to twin images. Note, however, that this also proves that model calculations based on the results obtained from few energies can be used for a detailed refined structural analysis. In addition, a larger number of counts reduces the experimental errors and makes a more detailed analysis of the peak shapes reasonable. All this will, in the future, increase the precision of the measurements considerably.

\section{SUMMARY AND CONCLUSION}

A bcc Fe single crystal served as test structure in the first realization of pure $\mathrm{x}$-ray-fluorescence holography to assess the precision and limitation of XFH. Pure measurements ensure that a straightforward data evaluation and interpretation becomes possible. This is especially important for future applications of the method to unknown structures, where a mixing of direct and reciprocal signals has to be avoided. The shift of atom positions in the reconstructed images was shown to be due to the limited energy range available in direct x-ray-fluorescence holography but may be overcome by reciprocal holography experiments or by refined model calculations. A three-dimensional reconstruction of the $\mathrm{Fe}$ bcc structure with isotropic high spatial resolution was obtained by using the symmetry information from the Kossel lines to extend the hologram to the full $4 \pi$ angular range. Pure XFH can be measured in a large angular range and will make a nearly isotropic high spatial resolution possible even for noncrystalline structures where no symmetry information from Kossel or XSW patterns is available. Future x-ray freeelectron lasers using the excitation principle of self-amplified spontaneous emission ${ }^{23-26}$ can provide high brilliances in one 100 fs long x-ray flash. XFH with such a source will make ultrafast time-resolved atomic resolution holography possible. 
${ }^{1}$ M. Tegze and G. Faigel, Nature (London) 380, 49 (1996).

${ }^{2}$ T. Gog, P.M. Len, G. Materlik, D. Bahr, C.S. Fadley, and C. Sanchez-Hanke, Phys. Rev. Lett. 76, 3132 (1996).

${ }^{3}$ A. Szöke, in Short Wavelength Coherent Radiation: Generation and Applications, edited by D. T. Attwood and J. Bokor, AIP Conf. Proc. 147 (AIP, New York, 1986), p. 361.

${ }^{4}$ T. Gog, D. Bahr, and G. Materlik, Phys. Rev. B 51, 6761 (1995).

${ }^{5}$ D.V. Novikov, B. Adams, T. Hiort, E. Kossel, G. Materlik, R. Menk, and A. Walenta, J. Synchrotron Radiat. 5, 315 (1998).

${ }^{6}$ B. Adams, D.V. Novikov, T. Hiort, G. Materlik, and E. Kossel, Phys. Rev. B 57, 7526 (1998).

${ }^{7}$ B. Adams, Y. Nishino, and G. Materlik (unpublished).

${ }^{8}$ B. Adams, T. Hiort, E. Kossel, G. Materlik, Y. Nishino, and D.V. Novikov, Phys. Status Solidi B 215, 757 (1999).

${ }^{9}$ T. Gog, R.H. Menk, F. Arfelli, P.M. Len, C.S. Fadley, and G. Materlik, Synchrotron Radiation News 9, 30 (1996).

${ }^{10}$ T. Gog, R.A. Eisenhower, R.H. Menk, M. Tegze, and G. LeDuc, J. Electron Spectrosc. Relat. Phenom. 92, 123 (1998).

${ }^{11}$ J. Kawai, K. Hayashi, T. Yamamoto, S. Hayakawa, and Y. Gohshi, Anal. Sci. 14, 903 (1998).

${ }^{12}$ K. Hayashi, T. Yamamoto, J. Kawai, M. Suzuki, S. Goto, S. Hayakawa, K. Sakurai, and Y. Gohshi, Anal. Sci. 14, 987 (1998).
${ }^{13}$ M. Tegze, G. Faigel, S. Marchesini, M. Belakhovsky, and A.I. Chumakov, Phys. Rev. Lett. 82, 4847 (1999).

${ }^{14}$ G. Faigel and M. Tegze, Rep. Prog. Phys. 62, 355 (1999).

${ }^{15}$ R. Frahm, J. Weigelt, G. Meyer, and G. Materlik, Rev. Sci. Instrum. 66, 1677 (1995).

${ }^{16}$ R. W. G. Wyckoff, Crystal Structures, 2nd ed. (Interscience, New York, 1963), Vol. 1.

${ }^{17}$ J.J. Barton, Phys. Rev. Lett. 67, 3106 (1991).

${ }^{18}$ P.M. Len, S. Thevuthasan, C.S. Fadley, A.P. Kaduwela, and M.A. Van Hove, Phys. Rev. B 50, 11275 (1994).

${ }^{19}$ W. Kossel, H. Loeck, and H. Voges, Z. Phys. 94, 139 (1935).

${ }^{20}$ J.J. Barton, Phys. Rev. Lett. 61, 1356 (1988).

${ }^{21}$ B.P. Tonner, Zhi-Lan Han, G.R. Harp, and D.K. Saldin, Phys. Rev. B 43, 14423 (1991).

${ }^{22}$ D.K. Saldin, G.R. Harp, B.L. Chen, and B.P. Tonner, Phys. Rev. B 44, 2480 (1991).

${ }^{23}$ A.M. Kondratenko and E.L. Saldin, Part. Accel. 10, 207 (1980)

${ }^{24}$ R. Bonifacio, C. Pellegrini, and I.M. Narducci, Opt. Commun. 50, 373 (1984)

${ }^{25}$ J. Arthur, G. Materlik, R. Tatchyn, and H. Winick, Rev. Sci. Instrum. 66, 1987 (1995).

${ }^{26}$ R. Brinkmann, G. Materlik, J. Rossbach, J.R. Schneider, and B.-H. Wiik, Nucl. Instrum. Methods Phys. Res. A 393, 86 (1997). 